\title{
Acceptability of screening for early detection of liver disease in hazardous/harmful drinkers in primary care
}

\begin{abstract}
Background

It is estimated that one-quarter of adults in the UK drink at harmful/hazardous levels leading to increased mortality and alcohol liver disease (ALD). The Alcohol Liver Disease Detection Study (ALDDeS) aimed to test out in primary care the feasibility of alcohol misuse screening in adults, using the AUDIT questionnaire, and to assess screening harmful/hazardous alcoho users for ALD using newer non-invasive serum markers of fibrosis.
\end{abstract}

\section{Aim}

To explore patients' experiences of taking part in ALDDeS and understanding of the delivery and process of screening for ALD using selfreport questionnaires and feedback of liver

fibrosis risk using levels of non-invasive serum markers.

\section{Design and setting}

A nested qualitative study based in five primary care practices in the UK.

\section{Method}

From a sample of patients who were identified as drinking at harmful/hazardous levels, 30 participants were identified by maximum variation sampling for qualitative in-depth interviews. Using the principles of constant comparison the transcribed interviews were thematically analysed.

\section{Results}

Receiving a postal AUDIT questionnaire was viewed as acceptable by participants. For some completing the AUDIT increased awareness of their hazardous alcohol use and a positive blood test indicating liver fibrosis was a

catalyst for behaviour change. For others, a negative blood test result provided a licence to continue drinking at hazardous levels. A limited understanding of safe drinking and of ALD was common.

\section{Conclusion}

Educational and training needs of primary care professionals must be taken into account, so that patients with marker levels indicating low risk of fibrosis are correctly informed about the likely risks of continuing to drink at the same levels.

\section{Keywords}

alcoholic liver disease; patient acceptance of health care; primary care; understanding.

\section{INTRODUCTION}

Alcohol misuse is a major public health problem in Europe and in the UK, an estimated $24 \%$ of adults drink hazardously or harmfully, ${ }^{1}$ costing the NHS in excess of $€ 2.7$ billion per annum. ${ }^{2}$ Problems associated with excess alcohol consumption include social issues, ${ }^{3}$ increased accidents, chronic health problems ${ }^{4}$ and mortality. In 2005 an estimated 14982 deaths were attributable to alcohol consumption and a high proportion of these were from alcohol liver disease (ALD).

ALD develops slowly with few symptoms at an early stage with patients often presenting with features of advanced liver disease. Compared to routine liver function tests newer serum markers of fibrosis have greater accuracy for detecting fibrosis or cirrhosis at an early stage. ${ }^{6}$ Such tests could be used along with alcohol screening to improve detection of liver disease in asymptomatic individuals with the potential to reduce the complications and mortality associated with severe liver disease. If high risk drinking patterns can be identified, brief alcohol interventions provide an effective and cost-effective approach to reducing consumption. ${ }^{7-9}$ The main setting for detecting those at risk and intervening is primary care ${ }^{10}$ with the attitudes and involvement of GPs as key factors in the

C Eyles, PhD, research fellow; M Moore, MSc, MRCP, FRCGP, reader in primary care research; W O'Brien, RGN, senior research sister; GM Leydon, BA, MA, PhD, Dip.PH reader in health research \& NIHR fellow University of Southampton, Primary Care and Population Sciences, Aldermoor Health Centre Southampton. N Sheron, MB, MD, FRCP, senior lecturer in medicine and clinical lead in hepatology, University of Southampton, Faculty of Medicine, Clinical and Experimental Sciences Academic Unit, University Hospital Southampton NHS Foundation Trust, Southampton. P Roderick MD, head of Primary Care and Population Sciences, University of Southampton, Faculty of Medicine, Primary Care and Population Sciences, success of brief alcohol interventions.11,12 However many barriers to implementing brief alcohol interventions in primary care exist in Europe ${ }^{13}$ and the UK ${ }^{14,15}$ with GPs reporting a lack of time in consultations, support and training, 12,13,15 and fears that discussing alcohol consumption will cause offence. ${ }^{16}$

A survey of patients' attitudes towards screening for 'at risk' drinking with selfreport questionnaires and an alcohol biomarker blood test showed that patients are in favour of these techniques being used. ${ }^{17}$ Patients also saw value in discussing alcohol consumption in primary care with a GP or health professional with whom they had developed a rapport, ${ }^{18,19}$ particularly if discussions are combined with lifestyle issues or when linked to other health conditions ${ }^{18,19}$ and as part of routine questioning in a consultation. ${ }^{20}$ However there is limited understanding of patients' experiences of screening for high risk drinking patterns using self-report questionnaires, blood tests, and the use of brief alcohol interventions in primary care. As outlined above, an understanding of the perceptions and barriers to alcohol interventions is essential for subsequent successful implementation. ALDDeS is a primary care based feasibility study, ${ }^{21}$ which aimed to test out population screening

Southampton General Hospital, Southampton. Address for correspondence

Caroline Eyles, University of Southampton, Primary Care and Population Sciences, Aldermoor Health Centre, Aldermoor Close, Southampton, S016 5ST.

E-mail: cgedsoton.ac.uk

Submitted: 7 December 2012; Editor's response: 4 February 2013; final acceptance: 3 April 2013. CBritish Journal of General Practice

This is the full-length article lpublished online 29 Jul 2013) of an abridged version published in print. Cite this article as: $\mathbf{B r} \mathbf{J}$ Gen Pract 2013; DOI: 10.3399/bjgp13X670642 


\section{How this fits in}

Patients when surveyed have reported the acceptability of possible blood test screening for alcohol liver disease in primary care. In this study patients reported that blood test screening in primary care was an acceptable process, and a positive blood test result can be a catalyst for drinking behaviour change. However a negative blood test could provide inappropriate reassurance, suggesting a great deal of care is required when screening. All interviewees were screened to be drinking at harmful/ hazardous levels and all conveyed uncertainty about national guidance on safe drinking and how such guidance relates to them as individuals.

for adults for alcohol misuse using selfreport questionnaires and liver fibrosis blood tests. This paper reports on a nested qualitative study, which was conducted with the aim of exploring patients' experiences and understanding about the delivery and process of screening and brief alcohol intervention in primary care.

\section{METHOD}

\section{Setting and participants}

The sample were selected from the group of patients who had tested as harmful/ hazardous drinkers (AUDIT >8). Patients were chosen using purposive maximum variation sampling to ensure heterogeneity of age, sex, locality lusing surgeries, from inner city to rural market town practices) and blood test results. Patients were selected from the four groups of hazardous drinkers that were originally identified in ALDDeS, as blood test: negative; borderline; positive; and strongly positive, (the grading of liver risk evolved during the main study into The Southampton Traffic Light test $(S T L)^{21}$ but was not relevant to this patient interview sample.

\section{Semi-structured interviews}

Patients were written to by the study team and invited to participate in a qualitative interview. On return of a reply slip indicating interest in participating, the patients were contacted to arrange an interview at their surgery. All interviews were conducted and analysed by one author. Semi-structured interviews permitted in-depth exploration of participants' experiences of taking part in the ALDDeS and views on alcohol in general. ${ }^{22}$ The interview guide (Box 1) was developed by the study team by consensus and assisted exploration of topics but was sufficiently flexible to allow participants to raise issues that were important to them. The interview guide was piloted with the first participant and no changes were made as a result. Consent was taken face-to-face before the interviews, which were held at the patient's surgery, lasted up to 60 minutes and were audiorecorded and transcribed verbatim in preparation for thematic analysis. Recruitment of patients continued until saturation of data was achieved (when no new codes/themes could be identified), it was estimated that 25-30 interviews would be sufficient to achieve saturation. ${ }^{22}$

\section{Analysis}

Analysis of the transcribed interviews started as soon as data collection commenced. Using constant comparison, whereby data are systematically compared within and between interviews, ${ }^{23}$ the transcripts were coded and themes and relationships between themes developed ${ }^{24}$ allowing the main issues for participants to be identified. Eight members of the ALDDeS team lconsisting of a mix of qualitative and quantitative researchers) analysed three transcripts each to check the quality of analysis, the validity of codes and themes, the consistency of their allocation and discrepancies. Any subsequent changes (such as new codes) were agreed by consensus.

\section{Box 1. Interview guide}

Part 1. Experiences of taking part in the ALDDeS study

- How would you describe your overall experience of taking part in the study?

- The acceptability of screening questionnaires (AUDIT).

- Being invited for and having a blood test.

Part 2. Your views and understanding of alcohol and safe drinking

- Tell me about you and alcohol and the role it has played in your life before you took part in this study.

- Tell me about you and alcohol and the role that it plays in your life after having taken part in the study.

- Before you took part in the study what did you know or understand about alcohol liver disease?

- How has your knowledge or understanding changed since taking part?

- What do you think constitutes safe drinking? 


\begin{tabular}{|c|c|}
\hline $\begin{array}{c}\text { Total } \\
\text { I }\end{array}$ & $\begin{array}{l}n=30 \text { ) } \\
n=4 \text { s }\end{array}$ \\
\hline \multicolumn{2}{|l|}{ Surgery } \\
\hline A Market town & 2 \\
\hline B Urban & 7 \\
\hline C Market town & 10 \\
\hline D Urban & 2 \\
\hline E Rural & 9 \\
\hline \multicolumn{2}{|l|}{ Sex } \\
\hline Female & 14 \\
\hline Male & 16 \\
\hline \multicolumn{2}{|l|}{ Age, years } \\
\hline $25-34$ & 3 \\
\hline $35-44$ & 14 \\
\hline $45-54$ & 13 \\
\hline \multicolumn{2}{|l|}{ Blood test } \\
\hline Did not attend clinic laudit >8) & 10 \\
\hline Negative & 8 \\
\hline Borderline positive & 3 \\
\hline Positive & 5 \\
\hline Strongly positive & 4 \\
\hline
\end{tabular}

\section{RESULTS}

Over a 4-month period 210 patients were contacted about participating in an interview, of these, 48 responded expressing an interest in the study. Thirty interviews were conducted and recruitment stopped at this point as saturation of the data was reached between interview 27 and 30 (Table 1). Three main themes emerged and are discussed; interview number, sex, age, and blood test result are provided after the quotations.

\section{Theme 1. Acceptability of the process of the study}

Being invited to complete a questionnaire about alcohol intake through a postal approach was low cost but carried the risk of not being acceptable to participants. Indeed, reactions to receiving the questionnaire were mixed. Some participants reported that participation was an opportunity to have a free health check. Others, who had concerns over their health, described the questionnaire as arriving at the 'right time', as it helped to trigger awareness of the levels of their alcohol consumption:

When I started filling out the questionnaire which talked about the volumes [of alcohol] ... that you're drinking ... It's the first sort of time for a long time ... you sort of ... actually sat down and worked out ... what it is you drink ... and when ... and I could ... I'd recognised that ... there was an issue there.' (Int. 7, male, age 44 years, blood test positive)

However, some were also curious or suspicious as to why they were selected, despite being informed that they were randomly selected:

'I felt maybe it was perhaps pointed just at me. I had no problem with it but that was my initial thought when I actually opened it. I thought, hum, why is it coming to me? You know. I thought mmm ... why, you know, a little bit suspicious of why me. But I had no problem with it.' (Int. 12, female, age 50 years, blood test positivel

Initial suspicion at being selected did not seem to preclude participation and this was often attributed to a growing concern about their health. Some described experiencing ill health while aware that they were drinking too much, while for others a family history of alcohol-related problems placed the topic of alcohol high on their own personal health agenda:
I have got a brother who's an alcoholic ... as well ... so ... you know ... there's that ... and I mean, it could be familial thing ...the fact that mine has come back slightly positive ... So I thought ... well ... you know ... I don't want to turn up ... like my brother ... he's in hospital at the moment ... so, I mean ... he's not in a good state of health with it ... um ... and you know... it's gone on for some years ... stopping and starting and ... I think my granddad died of ... liver cancer ... and I think he liked ... I think he liked his ... I think he was a fairly ... fairly heavy drinker.' (Int. 3 , female, age 55 years, blood test strongly positive).

All of the interviewed participants who attended for a blood test deemed this type of general practice-led screening process to be acceptable and some expressed a belief that the blood test results provided useful concrete 'diagnostic' evidence:

I think ... yeah ... many people are sort of interested ... particularly when it comes to sort of having ... tests ... done. An actual result ... um ... from it. It's like they do the ... um ... what's it the ... err ... sort of stool samples aren't they at the moment for ... sort of cancer? Yeah ... it's ... you know ... people ... err ... people like that sort of thing. [Laughs] ... Sounds odd doesn't it? We don't want to be lectured at ... but you know ... if we see ... some sort of hard evidence of ... sort of problems then ... then you know ... I think people do respond to that.' (Int. 5, male, age 39 years, blood test borderline positive)

\section{Theme 2. Alcohol blood testing: a catalyst for change?}

Six of the eight participants interviewed who had received a negative blood test reported that they did not intend to change their drinking habits. These participants appeared to be lulled into a false sense of security as a negative blood test was viewed as an indication that they could safely continue drinking at their pre-test levels, despite having received a brief alcohol intervention leaflet:

I would say my drinking has remained the same. I want or I wanted to start to be able to curb my drinking but that hasn't been the effect of being involved in this study, so far. And that may very well be because I had a negative blood test. So in a way it's given me a license to carry on behaving as I do because I'm getting away with it, at the moment.' 'Int. 27, female, age 45 years, blood test negative) 
Most of the participants who had positive blood tests reported that taking part in the ALDDeS study had either reinforced previous decisions to reduce their alcohol intake or stimulated them to reduce their alcohol intake:

'I found it ... I've ... Well ... for me ... it's given me a kick up the backside with my drinking ... I mean I'm not going to ... I'm not going to say that it will be easy to stop drinking. This is where the ... this is where the alcoholism side of it comes in ... as opposed to ... but now l'm just so used to drinking ... That ...it's part of my routine. To get it out of my routine ... is going to be difficult, but it is urgent that I get it out of my ... system and because of the ... the ... the findings of the study ..., uh, it's put me ... in short, sharp shock mode to do something about it.' (Int. 1, male, age 27 years, blood test strongly positive)

However, for some, this potential catalyst for change was mitigated by other factors such as uncertainty as to the meaning of the blood test results, skepticism as to the validity and relevance of their result:

'They, kind of ... they were a little inconclusive so I was left with ... a little bit ... unclear ... as to ... The test showed there was signs of ... um ... early signs of liver damage ... which could've been caused by a whole multitude of things and ... you know ... I was left quite clear that ... you know ... unless they did further tests ... it was impossible to say whether this was a hereditary thing ... you know ... through alcohol ... through taking painkillers ... or ... a multitude ...of different things ...' (Int. 6, male, age 44 years, blood test borderline positivel

For some participants the GPs attempt to allay fears or to clarify a situation seemed instead to create confusion, especially regarding the necessity or otherwise to change their drinking behaviour. In some cases this was perceived as due to insufficient advice given or advice that conflicted with study guidance:

'I didn't understand ... what is the difference between that test and what test my GP does ... you know ... quite regularly and ... So ... that ... So I came back to the GP ... and she said ... you know ... you need to cut down on the amount ... you drink ... Not to stop ... just ... follow the guidelines ... and she referred me for some more blood tests ... which I've had ... and they say it's now within normal limits ... so I don't know if it's the same blood test or a different blood test and that's where I'm confused. ... She wasn't worried that I was an alcoholic ...She ... you know ... just aware that I drink all my units on a Friday and a Saturday night, so it's classed as binge drinking ... So she wasn't worried ... which to me goes against what the letter said ... that I have cirrhosis and fibrosis. 'Int. 4 , female, age 37 years, blood test strongly positive)

Theme 3. Knowledge of safe drinking and alcohol liver disease (ALD) and its role in behaviour change

Most participants conveyed a limited understanding of safe drinking levels and expressed scepticism and/or confusion when asked about safe drinking guidelines:

'I don't know I mean, I would go out and binge drink till I was ... being sick and falling over but I didn't know I was drinking enough to cause me damage ... and I don't know, I mean ... units ... I'm not sure what they mean, are they on the label? I don't think any of us know what that means ... (Int. 12, female, age 50 years, blood test positive)

Some participants used individual factors to define whether their level of drinking was safe, such as a lack of a hangover or the ability to function at work. Some described the importance of using tangible physical effects of drinking alcohol as a measure of safe drinking, such as the ability to exercise, not feeling excessively tired and maintaining a healthy weight:

My body tells me when I've had too much ... horrible taste in your mouth ... Tummy feels funny and feel a little bit grumpy ... Definitely grumpy ... um ... I get grumpy with my ... little girl sometimes ... and I know it's because ... I've overdone ... the drink ... you know ... overdone ... It makes me feel muzzy and horrible ... but I don't like to think about the units because I think if you added them all up it would be a bit scary. ' (Int. 19, female, age 54 years, blood test negative)

Participants' knowledge of the health consequences of drinking was limited. Two nurse qualified participants and participants who had experienced illness of family or friends due to excess alcohol consumption discussed the subject with some confidence. However, most asserted their knowledge in rather equivocal terms and described how they had acquired knowledge of ALD through popular media: 
I mean / heard a radio programme a number of years' ago now, about George Best and a liver doctor said well if you give up for a day a week, it's fine, because your liver will renew yourself, so you always do that, you'll always be fine. And so I happily believed that for quite a few years.' (Int. 11, female, age 50 years, blood test positive)

Many participants who had the blood test reported that ALDDeS increased knowledge of safe drinking guidelines and consequently increased their awareness of their own alcohol intake, as well as the alcohol intake of others, including friends and family. This knowledge was acquired either through study literature, interaction with the study nurse or through personal research triggered by their participation. These participants described modifying their alcohol consumption using a number of self-management strategies such as making lifestyle changes and enlisting support of family and friends:

'You know, I haven't given up. Its knowing how to change ... no one has suggested ways ... I think ... It had become more of a habit I think ... of coming in and...you know ... you cook tea and then you have a glass of wine with tea ... But um ... you know ...now I do quite a bit of sport ... You know ... I don't necessarily drink every night ... Or if I go out. I play bridge one night and I know ... I drive ... so I don't ... you know, I wouldn't have drink then ... sometimes / offer to drive just so I don't have a drink.' (Int. 3, female, age 55 years, blood test strongly positive)

\section{DISCUSSION}

\section{Summary}

\section{Ethical approval}

Ethical approval was granted by Southampton \& South West Hampshire Research Ethics Committee on 10 October 2007 (REC reference 07/H0502/160).

\section{Provenance}

Freely submitted, externally peer reviewed.

\section{Competing interests}

The authors have declared no competing interests.

\section{Acknowledgements}

We thank the practices who took part in the study, the patients who agreed to be interviewed and our lay advisor who contributed to data interpretation.

\section{Discuss this article}

Contribute and read comments about this article on the Discussion Forum: http://www.rcgp.org.uk/bjgp-discuss of alcohol consumption, however, a better understanding was described by participants who were health professionals or who had experienced illness of family and/or friends due to excess alcohol consumption.

\section{Strengths and limitations}

Qualitative interviews were the optimal method of data collection. However, as with all interview studies the kind of data generated is limited to the perspective of a self-selected group of participants. Nevertheless, this perspective offers insight into experiences that can help to explain findings from larger studies of the early detection of liver disease and help to inform policy change. Interviews to explore GPs' perspectives on the impact of the study within their practices and to explore how they explained test results to their patients would have provided a useful complement to patients' interviews. There was less availability of participants within the 25-40-year old age group and this limitation is reflected in the study sample for this qualitative study. Despite the modest sample of 30 interviewees analytic saturation was reached and many perspectives discussed resonate with other relevant studies thereby increasing confidence in the face validity and transferability of the findings.

\section{Comparison with existing literature}

This study echoes a previous study which showed that completing an AUDIT can result in increased awareness of alcohol consumption, ${ }^{25}$ and although initially sceptical, patients had positive opinions about self-report alcohol screening and the use of blood tests. ${ }^{26,27}$ Adding to this evidence it is suggested that patients may make changes as a consequence of a positive blood test. This suggests that the feedback of test results that clearly signal bodily harm attributable to drinking behaviour may increase motivation to change; ${ }^{28}$ the significance of the results are likely to be instantly understandable and concerning. ${ }^{29}$ This corresponds with what is known from smoking cessation studies. ${ }^{30-32}$

In ALDDeS a high proportion of participants reduced their drinking in comparison to previous studies of brief alcohol interventions. ${ }^{21}$ It is possible that some of the participants interviewed in the negative test group derived a sense of false reassurance from a negative result reinforcing an already existing resistance to change. Indeed in such cases it might be that a negative blood test may require 
more explanation to the participants in the context of their high AUDIT scores. The STL was a new test and GPs in the study were not familiar with its use. It was not made clear that although the STL test is reasonably good at identifying moderate and severe liver fibrosis, it will miss cases of very early fibrosis, and it is critical that patients are informed that a negative test result (or green traffic light) is not a 'green light' for continued drinking, but a sign that the problem has been caught in time. This message clearly did not get across in all cases, and this will need to be addressed in future studies.

Nationally, knowledge about safe drinking guidelines is (slowly) improving. ${ }^{33}$ It is possible that such confusion has arisen, in part, from changes in published guidelines on weekly drinking limits to daily limits $^{34,35}$ and that sentiment was echoed by some participants. Adding to the confusion is the growing awareness of the role of binge or episodic drinking in ALD, 36,37 which contradicts other reports that it is a steady pattern of drinking daily or near daily heavy drinking which is particularly hazardous. ${ }^{38}$ This is likely compounded by confused messages in the popular media ${ }^{39}$ and a dearth of widespread and consistent GP-based education and counselling. ${ }^{40}$

Possibly arising from this confusion, participants described the difficulty of knowing what they were meant to adhere to in terms of safe drinking, relying instead on very individual definitional criteria and safe drinking guidance grounded in their own beliefs and experiences, and this has been noted elsewhere. ${ }^{41}$ This suggests that there is a need for a consistent, coherent and understandable policy on safe drinking.

\section{Implications for research and practice}

There is robust evidence supporting the use of brief alcohol interventions in primary care: feedback of biomarkers have the potential to provide a catalyst for behaviour change. However, the information that is conveyed to participants particularly about their blood result is key to their understanding. A patient who has a negative blood test but who is still drinking at harmful/hazardous levels will need support in understanding the potential risks of continuing to drink. Information about risk can be presented differently depending on preferences ${ }^{42,43}$ and a more tailored approach for this particular group may be necessary. It is also important to take into account the educational and training needs of primary care health professionals, including information about newer fibrosis tests to ensure GPs are comfortable sharing appropriate feedback to patients. This work supports the need for a randomised controlled trial to evaluate the impact on primary care patients of feedback of liver fibrosis markers, taking into account the qualitative data and addressing the use of the 'green traffic light' terminology and the need for appropriate training and a consistent message. 


\section{REFERENCES}

1. Drummond CS, Oyefeso A, Phillips T. Alcohol Needs Assessment Research Project (ANARP). The 2004 national alcohol needs assessment for England. London: Department of Health, University of London St George's Division of Mental Health Section of Addictive Behaviour. http://www.alcohollearningcentre. org.uk/_library/Resources/ALC/OtherOrganisation/Alcohol_needs_assessment_ research_project.pdf laccessed 28 May 2013).

2. NICE. Alcohol use disorders: preventing the development of hazardous and harmful drinking. London: NICE, 2010. http://www.nice.org.uk/PH24 laccessed 28 May 2013).

3. Orford J. Coping with drug and alcohol problems: the experience of family members in three contrasting cultures. London: Routledge, 2005

4. Rehm J, Baliunas D, Borges GL, et al. The relation between different dimensions of alcohol consumption and burden of disease: an overview. Addiction 2010; 105(5): 817-843

5. Jones L, Bellis M, Dedham D. Alcohol-attributable fractions for England (alcohol-attributable mortality and hospital admissions). Liverpool: North West Public Health Observatory, 2008.

6. Gressner OA, Weiskirchen R, Gressner AM. Biomarkers of liver fibrosis: Clinical translation of molecular pathogenesis or based on liver-dependent malfunction tests. Clin Chim Acta 2007; 381(2): 107-113

7. Ashenden R, Silagy C, Weller D. A systematic review of the effectiveness of promoting lifestyle change in general practice. Fam Pract 1997; 14(2): 160-176.

8. Bertholet N, Daeppen JB, Wietlisbach V,et al. Reduction of alcohol consumption by brief alcohol intervention in primary care: systematic review and metaanalysis. Arch Intern Med 2005; 165(9): 986-995

9. Kaner E, Beyer F, Dickinson $\mathrm{H}$, et al. Effectiveness of brief alcohol interventions in primary care populations. Cochrane Database Syst Rev 2007; (2): CD004148.

10. Verrill C, Smith S, Sheron N. Are the opportunities to prevent alcohol related liver deaths in the UK in primary or secondary care? A retrospective clinical review and prospective interview study. Subst Abuse Treat Prev Policy 2006; 1: 16.

11. Kaner E, Rapley T, May C. Seeing through the glass darkly? A qualitative exploration of GPs' drinking and their alcohol intervention practices. Fam Pract 2006; 23(4): 481-487.

12. Wilson GB, Lock CA. Heather $\mathrm{N}$, et al. Intervention against excessive alcohol consumption in primary health care: a survey of GPs attitudes and practices in England 10 years on. Alcohol Alcohol 2011; 46(5): 570-577.

13. Drummond C, Gual A, Goos C, et al. Identifying the gap between need and intervention for alcohol use disorders in Europe. Addiction 2011; 106(Suppl 1): 31-36.

14. Nilsen P. Brief alcohol intervention-where to from here? Challenges remain for research and practice. Addiction 2010; 105(6): 954-959.

15. Johnson M, Jackson R, Guillaume L, et al. Barriers and facilitators to implementing screening and brief intervention for alcohol misuse: a systematic review of qualitative evidence. J Public Health 2011; 33(3): 412-421.

16. Rapley T, May C, Frances Kaner E. Still a difficult business? Negotiating alcoholrelated problems in general practice consultations. Soc Sci Med 2006; 63(9): 2418-2428.

17. Miller PM, Thomas SE, Mallin R. Patient attitudes towards self-report and biomarker alcohol screening by primary care physicians. Alcohol Alcohol 2006; 41(3): 306-310.

18. Lock CA. Alcohol and brief intervention in primary health care: what do patients think? Prim Health Care Res Dev 2004; 5(02): 162-178.

19. Hutchings D, Cassidy P, Dallolio E, et al. Implementing screening and brief alcohol interventions in primary care: views from both sides of the consultation. Prim Health Care Res Dev 2006; 7(03): 221-229.

20. Nilsen P, Bendtsen P, McCambridge J, et al. When is it appropriate to address patients' alcohol consumption in health care - national survey of views of the general population in Sweden. Addict Behav 2012; 37(11): 1211-1226.

21. Sheron N, Moore M, O'Brien W, et al. Feasibility of detection and intervention for alcohol related liver disease in the community: the ALDDeS study. $\mathrm{Br} J$ Gen Pract 2013: in press.

22. Patton $M Q$. Qualitative research and evaluation methods. London: Sage Publications, 2002

23. Glaser BG, Strauss AL. The discovery of grounded theory: strategies for qualitative research. New York, NY: Aldine de Gruyter, 1967.

24. Braun V, Clarke V. Using thematic analysis in psychology. Qual Res Psychol 2006; 3: 77-101

25. McCambridge J, Day M. Randomized controlled trial of the effects of completing the Alcohol Use Disorders Identification Test questionnaire on self-reported hazardous drinking. Addiction 2008; 103(2): 241-248.

26. Miller PM, Ornstein SM, Nietert PJ, Anton RF. Self-report and biomarker alcohol screening by primary care physicians: the need to translate research into guidelines and practice. Alcohol Alcohol 2004; 39(4): 325-328.

27. Miller PM, Ravenel MC, Shealy AE, Thomas S. Alcohol screening in dental patients: the prevalence of hazardous drinking and patients' attitudes about screening and advice. J Am Dent Assoc 2006; 137(12): 1692-1698.

28. McClure JB. Are biomarkers useful treatment aids for promoting health behavior change? An empirical review. Am J Prev Med 2002; 22(3): 200-207.

29. Hirschl M, Francesconi C, Chudik M, et al. Degree of atherosclerosis predicts short-term commitment for smoking cessation therapy. Prev Med 2004; 39(1): 142-146.

30. Bovet P, Perret F, Cornuz J, et al Improved smoking cessation in smokers given ultrasound photographs of their own atherosclerotic plaques. Prev Med 2002 34(2): 215-220.

31. Bize R, Burnand B, Mueller Y, et al. Biomedical risk assessment as an aid for smoking cessation. Cochrane Database Syst Rev 2009; (2): CD004705.

32. Parkes G, Greenhalgh T, Griffin M, Dent R. Effect on smoking quit rate of telling patients their lung age: the Step2quit randomised controlled trial. BMJ 2008; 336(7644): 598-600.

33. Lader D, Steel M. Drinking: adults, behaviour and knowledge in 2009. London: Office for National Statistics, 2009.

34. Department of Health. Health of the nation. London: DoH, 1992.

35. Department of Health. Sensible drinking: report of an inter-departmental working group. London: DoH, 1995.

36. Department of Health. Safe. Sensible. Social. The next steps in the National Alcohol Strategy. London: DoH, 2007. http://webarchive.nationalarchives. gov.uk/20130107105354/http://mww.dh.gov.uk/en/Publicationsandstatistics/ Publications/PublicationsPolicyandGuidance/DH_075218 laccessed 10 July 2013).

37. Hackley C, Gry-Howell A, Griffin C, et al. The discursive constitution of the UK alcohol problem in Safe, Sensible, Social: A discussion of policy implications. Drugs: Education, Prevention, and Policy 2008; 15(S1): 61-74.

38. Hatton J, Burton A, Nash H, et al. Drinking patterns, dependency and life-time drinking history in alcohol-related liver disease. Addiction 2009; 104(4): 587.

39. Engels RCME, Hermans R, van Baaren RB, et al. Alcohol Portrayal on Television Affects Actual Drinking Behaviour. Alcohol Alcohol 2009; 44(3): 244-249.

40. Das AK, Corrado OJ, Kyerematen E, et al. Do doctors understand alcohol units? Clin Med 2009; 9(6): 525-527.

41. Green CA, Polen MR, Janoff SL, et al. 'Not getting tanked': Definitions of moderate drinking and their health implications. Drug Alcohol Depend 2007; 86(2-3): 265-273.

42. Goyder E, Barratt A, Irwig LM. Telling people about screening programmes and screening test results: how can we do it better? J Med Screen 2000; 7(3): 123-126.

43. Murray E, Linke S, Harwood E, et al. Widening access to treatment for alcohol misuse: description and formative evaluation of an innovative web-based service in one primary care trust. Alcohol Alcohol 2012; 47(6): 697-701. 